\title{
Evaluation of a specialist cognitive clinic for the Deaf community
}

\author{
Authors: Matthew ] Harris, ${ }^{\mathrm{A}}$ Darica Au, ${ }^{\mathrm{B}}$ Katy Judd,,${ }^{\mathrm{C}}$ Joanna R Atkinson, ${ }^{\mathrm{D}}$ Maureen Bergson ${ }^{\mathrm{E}}$ and \\ Catherine J Mummery ${ }^{\mathrm{F}}$
}

There are significant health inequalities between Deaf and hearing people, including barriers to accessing care and communication difficulties in consultations. Such problems have particularly affected Deaf people with acquired cognitive deficits, leading to late and missed diagnoses. We therefore established a specialist cognitive clinic for the Deaf community in 2011 at the National Hospital for Neurology and Neurosurgery, which to our knowledge is the first of its kind in the world. In this study, we retrospectively analysed electronic patient records to evaluate the service and its impact since inception. We found that Deaf patients who use British sign language had difficulty obtaining an accurate diagnosis before attending our specialist clinic, highlighting the importance of tailored services for Deaf people. Our results show that the clinic improved communication for patients and accessibility to specialist investigations, ensuring diagnostic accuracy and overall reducing health inequality for this population.

KEYWORDS: Cognition, dementia, communication, health inequality, Deaf

DOI: 10.7861/clinmed.2020-1098

\section{Introduction}

In the UK there are over 87,000 people who are Deaf and use British Sign Language (BSL) as their first language.' This group has significant health inequalities compared to the hearing

Authors: ${ }^{A}$ Clinical research fellow, Dementia Research Centre, National Hospital for Neurology and Neurosurgery, London, UK; ${ }^{B}$ medical student, University College London Medical School, London, UK; ' Consultant nurse, Dementia Research Centre, National Hospital for Neurology and Neurosurgery, London, UK; ${ }^{D}$ Clinical neuropsychologist, Deafness, Cognition and Language Research Centre, University College London, London, UK; Eregistered sign language interpreter, Dementia Research Centre, National Hospital for Neurology and Neurosurgery, London, UK; ${ }^{\text {F }}$ Consultant neurologist, Dementia Research Centre, National Hospital for Neurology and Neurosurgery, London, UK population, including barriers to accessing healthcare, a lack of health information in accessible formats and poor communication during healthcare consultations. As a result, Deaf people have lower rates of diagnosis, are undertreated and have reduced awareness of common treatable conditions such as hypertension, diabetes and cardiovascular disease. ${ }^{2}$ Overall, the incorrect diagnosis and inadequate treatment of Deaf patients probably costs the NHS $£ 30$ million per year. ${ }^{3}$

Deaf people can encounter difficulties accessing healthcare in many areas of the NHS. They frequently report problems scheduling GP appointments, with many having to ask hearing relatives for help, or booking at the surgery in person. ${ }^{4}$ In secondary care, departments are reportedly unprepared for Deaf patients, with disjointed systems for booking appointments and communication support ${ }^{3}$ meaning that often BSL-English interpreters are not present for appointments, in particular for diagnostic services. There is also a relative lack of public health information provided in formats accessible to Deaf people (such as BSL), ${ }^{5}$ with a resultant reduced awareness of lifestyle factors important to prevent disease amongst Deaf people, ${ }^{6}$ and the potential for delayed presentation for medical assessment.

When Deaf patients are able to access healthcare, they often report communication difficulties, with some describing 'fear, mistrust and frustration', 7 and up to a third avoiding booking to see their GP because of such problems. ${ }^{5}$ Access to a qualified interpreter is a particular issue, with only $30 \%$ of Deaf patients reporting being able to communicate in BSL in GP consultations despite $80 \%$ wishing to do so. ${ }^{5}$ Instead, patients have to lipread or write notes in English, both of which can lead to misunderstanding, particularly because Deaf patients may not have good literacy or well-developed English skills. These issues can be further compounded by the use of unqualified interpreters ${ }^{8}$ and a lack of awareness of Deaf culture. ${ }^{9}$ Additionally, when a hearing third party (such as a family member) is present, patients may be excluded from discussions with medical professionals, disregarding their autonomy. ${ }^{8}$

One patient group particularly affected by the above issues are Deaf BSL users with acquired cognitive deficits, who have been found to have poor access to services with late and missed diagnoses. ${ }^{10}$ The cognitive assessment of Deaf patients poses unique challenges, particularly if practitioners are not used to communicating with Deaf people or aware of Deaf culture. Acquired cognitive disorders such as dementia can manifest in many ways, including affecting an individual's ability to sign. 
Patients may make subtle language errors such as changing the shape or articulation of a sign or use gestures when they cannot think of a specific sign. The clinical importance of such errors is unlikely to be communicated by sign language interpreters who are trained to relay meaning rather than form. In addition, conventional cognitive tests are not normalised or designed for use with Deaf people and could therefore lead to incorrect diagnosis

In order to address the above need, in August 2011 the Cognitive Disorders Service for Deaf BSL users was established at the National Hospital for Neurology and Neurosurgery. The clinic aims were to improve patient access and diagnostic accuracy, and subsequently to reduce known health inequalities in this population. Initially, the service was not funded to enable a regular clinic and the capacity to see patients was therefore limited. As awareness increased, greater numbers of referrals were received. In 2017, the clinic was centrally funded by NHS England as a national multidisciplinary service and is now run on a monthly basis.

The clinic consists of a hearing consultant neurologist with expertise in cognitive neurology, a Deaf neuropsychologist with an interest in dementia and expertise in the psycholinguistics of BSL, a hearing BSL interpreter with experience in complex cognitive consultations, and a hearing specialist nurse able to communicate in BSL. Importantly, the team are consistent for all appointments, enabling optimal assessment. Notably, family members and friends are not used to interpret as they lack training in the highly complex process of interpreting and need to be able to take part in clinical discussions. In comparison, in the 2019 national memory service audit, family members were required to interpret in $31 \%$ of cases who needed an interpreter. ${ }^{11}$

The NICE guidelines for dementia ${ }^{12}$ accentuate the need for care provision to be person-centred and the clinic is organised with this in mind. Patients are initially invited for neuropsychological assessment in BSL lasting a minimum of 1.5 hours on the morning of clinic review. The patient's case is then discussed among the multidisciplinary team before a focused assessment in the cognitive clinic lasting over 1 hour. During the consultation, the neuropsychologist monitors communication and provides real-time feedback. Features such as dysfluency, sign finding difficulty or semantic errors may indicate aphasia or apraxia or otherwise inform diagnostic workup. Following the consultation, the patient and those accompanying them spend up to 30 minutes separately with the specialist nurse who provides additional information specific to the diagnosis and directs them to services and support for patients with dementia.

Research into healthy deaf cognitive ageing resulted in the development of a new BSL cognitive screening test which for the first time allowed neuropsychological assessment directly in BSL rather than through interpreters. This test was subsequently validated through our clinic for the cognitive evaluation of Deaf patients, showing good sensitivity and specificity in detecting dementia. ${ }^{13}$ This test requires a high proficiency in BSL, however, to ensure it is administered and interpreted correctly. At present it has not been validated for use by clinicians who have no knowledge of sign language.

We sought to evaluate the specialist Deaf service to determine whether the aims of the clinic were being met, namely, to improve patient access and diagnostic accuracy and to reduce known health inequalities in this population. Results from the evaluation were to be used to inform future provision of care.

\section{Methods}

We retrospectively analysed electronic patient records from all patients attending the clinic between August 2011 (when the clinic was established) until December 2018. Electronic records included the original referral letter, subsequent clinic letters and all investigation results.

We wished to understand the clinical presentations of patients being referred to the clinic and whether there had been barriers to obtaining a diagnosis. We therefore collected patient gender, age at referral, presenting symptoms and duration, details of the referrer and prior diagnosis given. We also collected comorbidities, particularly noting risk factors for dementia such as hypertension, hypercholesterolaemia, diabetes, cardiac disease, smoking and depression. ${ }^{14}$

In order to assess whether the clinic was achieving its aim of diagnostic accuracy we referred to the NICE guidelines for dementia, ${ }^{12}$ which recommend structural imaging to rule out reversible causes of cognitive decline and to assist with subtype diagnosis. In addition, further specialised tests are to be considered in cases where the diagnosis is uncertain and knowing the dementia subtype would change management. We therefore collected details of investigations performed, as well as the initial working diagnosis, final diagnosis and treatment given.

All patients diagnosed with dementia should have their care plan reviewed at least once within 12 months. ${ }^{15}$ We expected that patients in this clinic would particularly benefit from follow-up compared to the hearing population due to limited dementia resources being available in BSL. We therefore collected data on the number of patients followed up and the duration.

This study was a service evaluation and therefore ethical approval was not required. As this is the first clinic of its kind, there is no gold standard for reference.

\section{Results}

36 patients ( 15 male, 21 female) were reviewed over the time period examined and the median age at referral to the clinic was 71.5 years (range $35-83$ ). All patients were referred with memory difficulties, with additional symptoms of behavioural change (31\%), communication difficulties $(6 \%)$ and abnormal movements (3\%). The median duration of symptoms prior to being seen in the clinic was 2 years (range 1-10 years) and the source of referrals was GP (81\%), consultant neurologist/psychiatrist (11\%) and psychologist ( $8 \%$ ). $17 \%$ of patients had previously been assessed by the local memory clinic. High levels of comorbidities were found in this patient group, as illustrated in Fig 1.

\section{Investigation}

All 36 patients were reviewed by a consultant neurologist with a qualified interpreter and underwent neuropsychological testing directly in BSL by a neuropsychologist. Cranial imaging (either CT or MRI head) was undertaken in $92 \%$ of patients. Local imaging was recommended for one patient who had difficulty travelling to clinic. Imaging was deemed not indicated for one patient, and one patient declined further investigation. Additional investigations included FDG-PET brain (8\%), DaTSCAN (3\%), lumbar puncture (25\%), EEG (17\%) and polysomnography (3\%). 


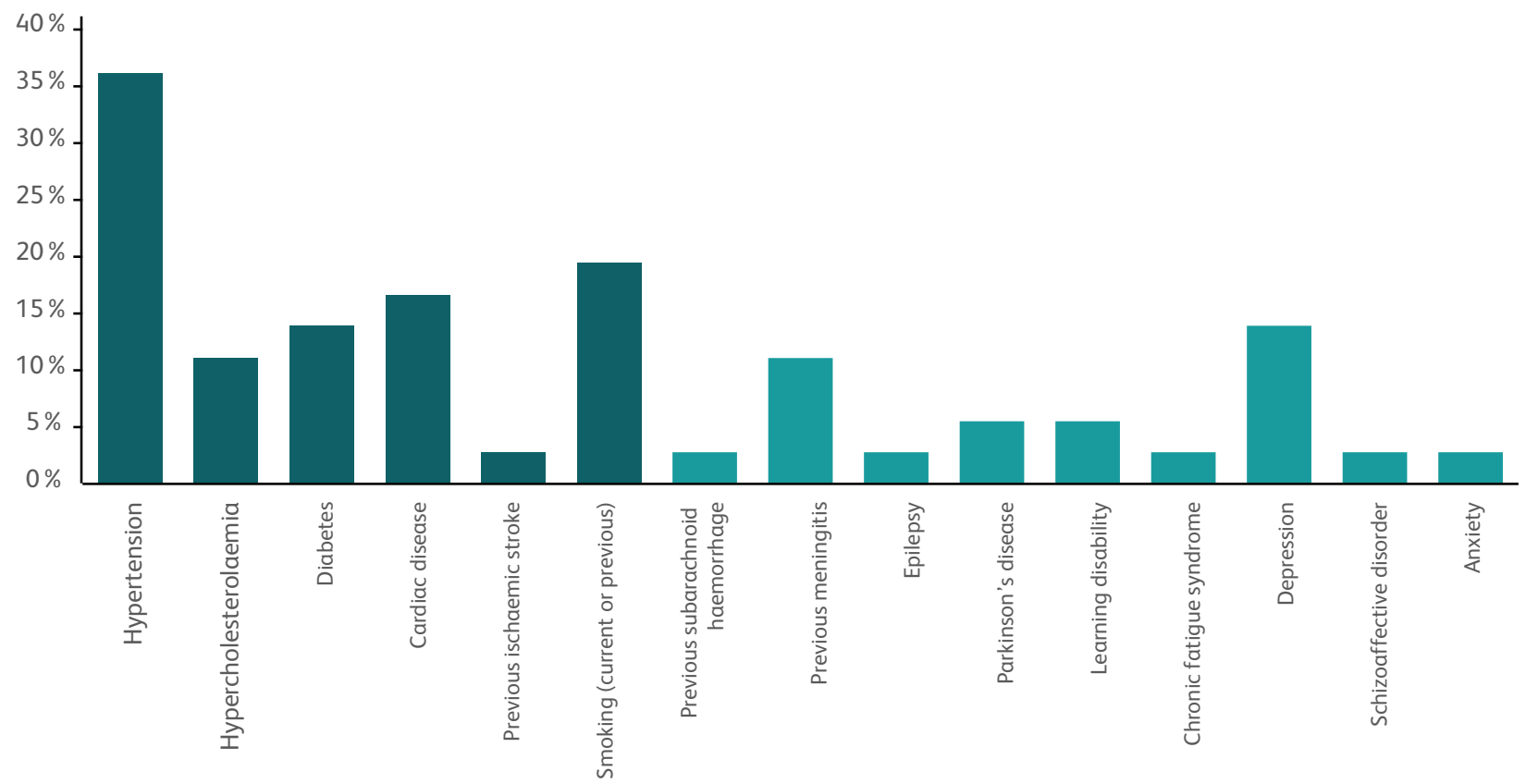

Fig 1. Bar chart showing the frequency of vascular (dark teal) and neurological/psychiatric (teal) comorbidities among patients referred to the clinic.

\section{Diagnosis}

The classification of all diagnoses made are displayed in Fig 2. $47 \%$ of patients attending the clinic were diagnosed with a neurodegenerative disorder and these are displayed in Fig 3. Primary psychological diagnoses included anxiety and depression. Other diagnoses included undiagnosed learning disability, prior encephalitis, probable mitochondrial disorder, obstructive sleep apnoea, periodic limb movements of sleep, insomnia, chronic pain and attentional memory difficulties.

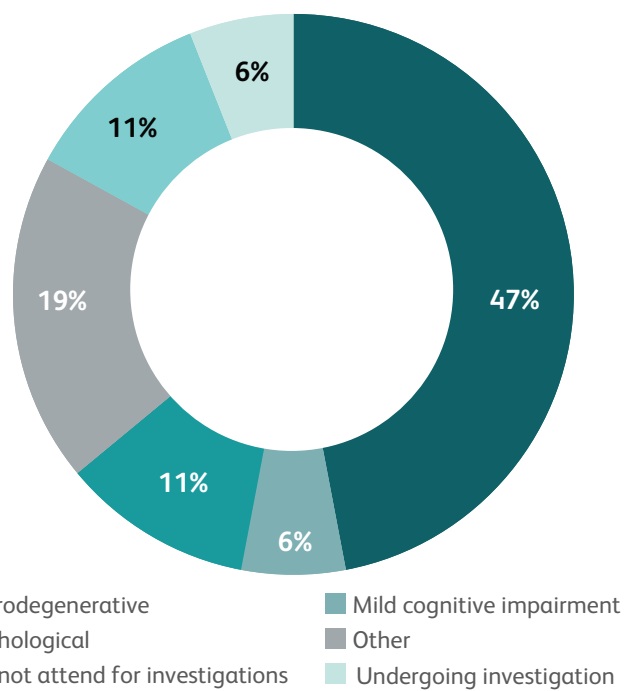

Fig 2. Classification of diagnoses made in clinic with corresponding percentage values.

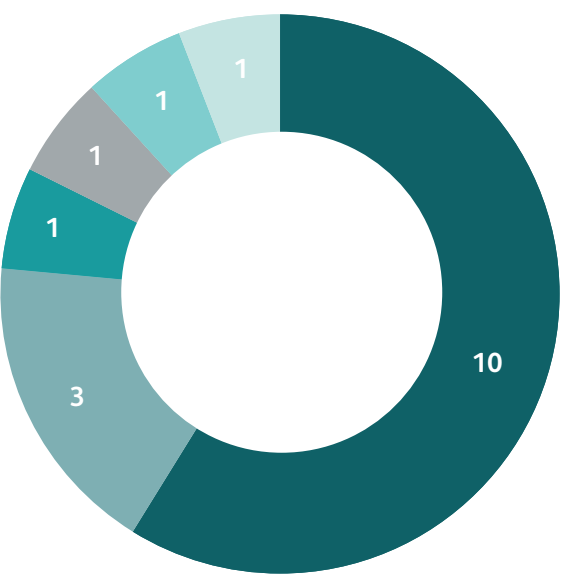

Typical Alzheimer's disease Logopenic aphasia secondary to Alzheimer's disease Vascular dementia PPA secondary to frontotemporal dementia

Parkinson's disease dementia Parkinson's disease MCI and small vessel disease

Fig 3. Breakdown of neurodegenerative diagnoses made, with number of patients indicated in each segment. MCI, mild cognitive impairment; PPA, primary progressive aphasia.

$83 \%$ of all patients referred did not have a prior diagnosis. In those with a prior diagnosis, this was changed in $67 \%$ of cases (four patients) following review in the specialist clinic, with three of these patients having been reviewed in a memory clinic previously. Importantly, two patients who were assessed as not having cognitive impairment prior to coming to clinic were subsequently diagnosed with mild cognitive impairment and Alzheimer's disease respectively. 


\section{Treatment}

All patients diagnosed with Alzheimer's disease were started on donepezil. Adverse effects were experienced by 3 (9\%) patients (headache, nausea, vomiting), leading to a reduction in dose or the patient being started on an alternative medication (rivastigmine or memantine). One patient with swallowing difficulty was changed to rivastigmine patches. In cases where psychological factors were thought to be contributory, one patient continued psychotherapy and one was referred for counselling. Other treatments included amitriptyline for chronic pain and insomnia; clonazepam for periodic limb movements of sleep; and changes to pre-existing antidepressant medication.

\section{Follow up}

One patient (3\%) who did not have dementia was discharged after initial review. All other patients were offered a follow-up appointment, of which 31 (86\%) patients attended. The median duration of follow-up was 17 months (range 3-70 months). Skype follow-up appointments were available for those unable to return to clinic.

Informal feedback from one relative stated: 'The clinic was a remarkably different experience for us. The team were fully Deaf aware. Not just at a surface level but they had deep understanding of what is normal and abnormal for a Deaf person... At last we were provided with an accurate neurological diagnosis.'

\section{Discussion}

In this evaluation, we found that Deaf patients with cognitive symptoms had difficulties obtaining an accurate diagnosis before attending the clinic, with long durations of symptoms prior to referral, cases of misdiagnosis, and longstanding undiagnosed conditions such as learning disability and prior encephalitis. These findings may in part be due to the fact that cognitive assessment can be challenging in this population due to language and cultural differences, with a lack of validated tests and norms. Missed or delayed diagnosis of dementia is also recognised to occur in the hearing population, particularly if the patient and clinician do not share a first spoken language or culture. 16,17 This is usually seen for complex or atypical dementia presentations, with young onset dementia patients reportedly waiting an average of 4.4 years for diagnosis. ${ }^{18}$ Our findings are therefore not unique to the Deaf community, but are indicative of the pervasive issue of inequitable access to dementia services, and highlight the importance of ensuring specialist services are accessible for all minority groups. ${ }^{19}$

We recognise the increased costs associated with running this clinic, particularly having access to both a BSL interpreter and a neuropsychologist fluent in BSL. Such measures ensure optimal communication however, and therefore diagnostic accuracy. In the hearing population, the use of professional interpreters has been shown to improve clinical care in those with limited English proficiency. ${ }^{20}$ It has also been suggested that any initial high costs from interpretation are offset by the long-term benefits, for example reducing the number of healthcare visits required and improving chronic disease outcomes. ${ }^{21}$

Patients referred to the clinic underwent thorough assessment by an expert team using validated cognitive tests alongside comprehensive investigation. In memory clinics across the UK, on average $11 \%$ of patients are referred for diagnostic neuropsychology, $85 \%$ deemed to require a brain scan, and access to specialist investigations (such as DAT scan, PET scan or CSF examination) can be variable, with only $2 \%$ of patients undergoing such testing. ${ }^{11}$ It is difficult to make significant comparisons between such clinics, however, due to the differing populations and individual patient needs. What is clear, is that Deaf patients in this clinic received a high-level service with neuropsychology and neuroimaging as routine for all patients.

High levels of comorbidities were found in this patient group with hypertension affecting $36 \%$ and diabetes in $14 \%$. By comparison, in the 2018 Health Survey for England, lower rates of hypertension (30\% in men and $26 \%$ in women) and diabetes ( $9 \%$ in men and $7 \%$ in women) were found in the general population, with similar smoking rates. ${ }^{22}$ The levels of comorbidity observed in our study are also comparable to rates previously reported in the Deaf community. ${ }^{2}$

A larger proportion of patients ( $53 \%$ overall) were diagnosed with a neurodegenerative disorder $(47 \%)$ or mild cognitive impairment $(6 \%)$ relative to other cognitive clinics (ranging from $30-45 \%)$. $^{23,24}$ Deaf people are recognised to have higher levels of risk factors for dementia, and one might therefore expect a higher prevalence in this cohort. There may have been fewer referrals of 'worried well' patients due to reduced awareness of dementia in the Deaf community. ${ }^{25}$ Even though there is significant coverage of dementia in the media and online, many older Deaf people do not have access to such resources in BSL. It is our experience that there may be a greater acceptance of cognitive diversity in the Deaf community, and widely held beliefs that a change in cognition is just linked to normal ageing. Deaf people may therefore delay seeking help for dementia until symptoms are at a more advanced stage.

A timely diagnosis is imperative for early intervention in dementia, but in this study some patients were experiencing symptoms for many years prior to referral. Barriers to accessing healthcare may have been contributory, ${ }^{26}$ with delayed cancer diagnosis reported in Deaf patients compared to the hearing population. ${ }^{27}$ Improved access to primary care and deaf awareness among GPs might therefore reduce times to referral. Furthermore, those with less education tend to take longer to seek help for cognitive symptoms, ${ }^{28}$ and Deaf people have lower average educational attainment compared to their peers due to a historical lack of fully accessible education. ${ }^{29}$ The provision of accessible resources in BSL on dementia and its symptoms, along with targeted publicity in BSL about the availability of specialist services for Deaf people, might also shorten the time to consultation and is a potential area for future action and research.

One limitation of our study is that we could not fully assess how easy it is for Deaf patients to obtain a referral to the clinic. As a tertiary provider, we could only record data on the patients actually referred. This is dependent on awareness of the service, patients being able to travel to the clinic, and whether the initial healthcare professional seeing the patient thinks specialist review is required. Based on a $1.3 \%$ prevalence of dementia in the general population, ${ }^{30}$ one would expect over 1,000 Deaf BSL users in the UK to have dementia. This number is likely to be an underestimate, however, as Deaf people have higher levels of modifiable risk factors for dementia such as obesity, hypertension, depression, lower education and social isolation. 2,14,29,31 Many more Deaf people might therefore benefit from both this specialist clinic and primary prevention strategies for dementia. 
In conclusion, our findings highlight the need for specialist services in providing healthcare for Deaf people who use British sign language and show that this clinic is achieving its aims of improving accessibility and communication, while reducing health inequality. In the future, we plan to increase awareness of the clinic through publicity and expand the number of slots available to allow more patients to be reviewed. We will also continue to evaluate and improve the service through patient feedback. To our knowledge, this is the first specialist cognitive clinic for Deaf BSL users in the world, and a step towards making the NHS goal of " $a$ personal, fair and diverse health and care system' a reality.

\section{References}

1 British Deaf Association. What is BSL? https://bda.org.uk/helpresources/\#BSL [Accessed 27 March 2021].

2 Emond A, Ridd M, Sutherland $\mathrm{H}$ et al. The current health of the signing Deaf community in the UK compared with the general population: a cross-sectional study. BMJ Open 2015;5:e006668.

3 SignHealth. A report into the health of Deaf people in the UK. SignHealth, 2014. https://signhealth.org.uk/wp-content/ uploads/2019/12/THE-HEALTH-OF-DEAF-PEOPLE-IN-THE-UK-.pdf [Accessed 27 March 2021].

4 Witte TN, Kuzel AJ. Elderly deaf patients' health care experiences. J Am Board Fam Pract 2000;13:17-22.

5 Pollard RQ, Dean RK, O'Hearn A, Haynes SL. Adapting health education material for deaf audiences. Rehabil Psychol 2009;54:23238.

6 Tamaskar P, Malia T, Stern C et al. Preventive attitudes and beliefs of deaf and hard-of-hearing individuals. Arch Fam Med 2000;9:518-25.

7 Steinberg AG, Barnett S, Meador HE, Wiggins EA, Zazove P. Health care system accessibility. Experiences and perceptions of deaf people. J Gen Intern Med 2006;21:260-6.

8 Action on Hearing Loss. Access all areas? A report into the experiences of people with hearing loss when accessing healthcare. RNID, 2013. https://rnid.org.uk/wp-content/uploads/2020/10/ Access-all-areas_Full-Repot_FINAL.pdf [Accessed 27 March 2021].

9 Lesch H, Burcher K, Wharton T, Chapple R, Chapple K. Barriers to healthcare services and supports for signing deaf older adults. Rehabil Psychol 2019;64:237-44.

10 Atkinson J, Woll B. The health of deaf people. Lancet 2012;379:2239.

11 Cook L, Souris H, Isaacs J. The 2019 national memory service audit. NHS England and Improvement, 2020. www.england.nhs.uk/ london/wp-content/uploads/sites/8/2020/04/The-2019-nationalmemory-service-audit.pdf [Accessed 27 March 2021].

12 National Institute for Health and Clinical Excellence. Dementia: assessment, management and support for people living with dementia and their carers. NICE guideline NG97. NICE, 2018 www.nice.org.uk/guidance/ng97 [Accessed 27 March 2021].

13 Atkinson J, Denmark T, Marshall J, Mummery C, Woll B. Detecting cognitive impairment and dementia in Deaf people: The British Sign Language Cognitive Screening Test. Arch Clin Neuropsychol 2015;30:674-711.

14 Livingston G, Huntley ], Sommerlad A et al. Dementia prevention, intervention, and care: 2020 report of the Lancet Commission. Lancet 2020:396:413-46.

15 National Collaborating Centre for Mental Health. The Dementia Care Pathway. Full implementation guidance. National
Collaborating Centre for Mental Health, 2018. www.rcpsych.ac.uk/ docs/default-source/improving-care/nccmh/dementia/nccmhdementia-care-pathway-full-implementation-guidance.pdf?sfvrsn=cdef189d_8 [Accessed 27 March 2021].

16 Bradford A, Kunik ME, Schulz P, Williams SP, Singh H. Missed and delayed diagnosis of dementia in primary care: prevalence and contributing factors. Alzheimer Dis Assoc Disord 2009;23:306-14.

17 De Moissac D, Bowen S. Impact of language barriers on quality of care and patient safety for official language minority francophones in Canada. J Patient Exp 2019;6:24-32.

18 Van Vliet D, de Vugt ME, Bakker C, Pijnenburg YA, Vernooij-Dassen MJ, Koopmans RT, Verhey FR. Time to diagnosis in young-onset dementia as compared with late-onset dementia. Psychol Med 2013:43:423-32.

19 UK Government. Equality Act 2010. Sections 20 and 29. London: 2010. www.legislation.gov.uk/ukpga/2010/15/contents [Accessed 27 March 2021].

20 Karliner LS, Jacobs EA, Chen AH, Mutha S. Do professional interpreters improve clinical care for patients with limited English proficiency? A systematic review of the literature. Health Serv Res 2007:42:727-54.

21 Bischoff A, Denhaerynck K. What do language barriers cost? An exploratory study among asylum seekers in Switzerland. BMC Health Serv Res 2010;10:248.

22 NHS Digital. Health Survey for England 2018. NHS Digital, 2018. https://digital.nhs.uk/data-and-information/publications/statistical/ health-survey-for-england/2018 [Accessed 27 March 2021].

23 Menon R, Larner AJ. Use of cognitive screening instruments in primary care: the impact of national dementia directives (NICE/SCIE, National Dementia Strategy). Fam Pract 2011;28:272-6.

24 Blackburn DJ, Shanks MF, Harkness KA, Reuber M, Venneri A. Political drive to screen for pre-dementia: not evidence based and ignores the harms of diagnosis. BMJ 2013;347:f5125.

25 Ferguson-Coleman E, Keady J, Young A. Dementia and the Deaf community: knowledge and service access. Aging Ment Health 2014;18:674-82.

26 Lesch H, Burcher K. Wharton T, Chapple R, Chapple K. Barriers to healthcare services and supports for signing deaf older adults. Rehabil Psychol 2019;64:237-44.

27 Druel V, Hayet H, Esman L et al. Assessment of cancers' diagnostic stage in a Deaf community - survey about 4363 Deaf patients recorded in French units. BMC Cancer 2018:18:93.

28 Zhao M, Lv X, Tuerxun M et al. Delayed help seeking behavior in dementia care: preliminary findings from the Clinical Pathway for Alzheimer's Disease in China (CPAD) study. Int Psychogeriatr 2016;28:211-19.

29 Hendar O, O'Neill R. Monitoring the achievement of deaf pupils in Sweden and Scotland: approaches and outcomes. Deafness Educ Int 2016;18:47-56.

30 Alzheimer's Society. Dementia UK: Second edition - Overview. Alzheimer's Society, 2014. http://eprints.lse.ac.uk/59437/1/Dementia_ UK_Second_edition_-_Overview.pdf [Accessed 27 March 2021].

31 Mackenzie I, Smith A. Deafness - the neglected and hidden disability. Ann Trop Med Parasitol 2009;103:565-71.

Address for correspondence: Dr Matthew Harris, Dementia Research Centre, Box 16, National Hospital for Neurology and Neurosurgery, Queen Square, London WC1N 3BG, UK. Email:mjharris@doctors.org.uk 